\title{
The counterregulating role of ACE2 and ACE2-mediated angiotensin 1-7 signaling against angiotensin II stimulation in vascular cells
}

\author{
Norihiro Hayashi, Koichi Yamamoto, Mitsuru Ohishi, Yuji Tatara, Yasushi Takeya, Atsushi Shiota, \\ Ryosuke Oguro, Yoshihiro Iwamoto, Masao Takeda and Hiromi Rakugi
}

To clarify the role of endogenous angiotensin (Ang)-converting enzyme 2 (ACE2) and its cleavage product, Ang 1-7, in the atherogenic stimulation of vascular cells, we investigated the effect of pharmacological inhibition of ACE2 and Mas, an Ang 1-7 receptor, on cellular responses against Ang II stimulation. We measured extracellular signal-regulated kinase (ERK) 1/2 phosphorylation by western blot, smooth muscle cell (SMC) proliferation by WST assay and the adhesion of monocytes labeled with PKH67 to endothelial cells (ECs) by fluorescence microplate reader. Cells were pretreated with Ang 1-7, olmesartan (Ang II type 1 receptor (AT1) blocker), DX600 (ACE2 inhibitor), D-Ala7-Ang1-7 (D-Ala; Mas antagonist), or combinations of treatments before the application of Ang II. Treatment with Ang II increased phosphorylated ERK 1/2 of SMC and EC, proliferation of SMC and adhesion of monocyte to EC, which were blocked by olmesartan. Pretreatment with DX600 either did not accelerate or only slightly accelerated these cellular responses. However, when Ang II signaling through AT1 was reduced by olmesartan, the additional treatment with DX600 significantly blunted some of the effect of olmesartan. Similarly, pretreatment with D-Ala reduced the inhibitory effect of olmesartan in response to Ang II stimulation. Endogenous ACE2 in vascular cells may contribute to counteracting the Ang II-mediated cellular response partly by upregulating the Ang 1-7 signaling through Mas. Hypertension Research (2010) 33, 1182-1185; doi:10.1038/hr.2010.147; published online 12 August 2010

Keywords: ACE2; adhesion; angiotensin 1-7; cell proliferation; Mas

\section{INTRODUCTION}

The proliferation of vascular smooth muscle cells (VSMCs) and the dysfunction and apoptosis of endothelial cells (ECs) characterize the initial stages of the atherosclerotic disease process. ${ }^{1}$ These pathophysiological changes in ECs and VSMCs are enhanced by an interaction with local autocrine, paracrine and intracrine factors, which promote local inflammatory reactions. Angiotensin II (Ang II), which is a major regulator of blood pressure by regulation of resistant vessels and sodium retention, is one of the most important factors involved in the promotion of the atherogenic response in vessel walls. Ang II binds to Ang II type 1 receptor (AT1) and exhibits atherogenic activities, such as cell proliferation or differentiation, through the activation of the extracellular-regulated kinase (ERK) $1 / 2 .^{2}$

Recently, the Ang-converting enzyme 2 (ACE2)-Ang 1-7-Mas axis has received researchers' attention as a counterregulator of the ACEAng II-AT1 axis. ACE2 is expressed in vascular cells, including VSMCs and ECs, and was reported to be upregulated in the aorta of the spontaneous hypertensive rat in response to Ang receptor blocker (ARB) treatment. ${ }^{3}$ In the renin-Ang system, ACE2 functions as a mono-coxypeptidase by mainly contributing to the generation of Ang
1-7 from Ang II, which shows a much higher catalytic efficiency than the conversion of Ang I to Ang 1-9. ${ }^{4}$ Many studies have shown the pivotal role of Ang 1-7 in the cardiovascular system by the exogenous administration of the peptide into mammalian cells or animals, ${ }^{5-8}$ including exerting an antiproliferative effect on $\mathrm{SMCs}^{8}$ and counterregulating of Ang II signaling in ECs. ${ }^{7}$ Recently, a G-protein-coupled receptor, Mas, was identified as a functional receptor of Ang 1-7.9 It has been reported that Mas-deficient mice with a $\mathrm{FVB} / \mathrm{N}$ genetic background exhibit both an elevation in blood pressure and endothelial dysfunction, suggesting that the physiological role of Ang 1-7 signaling in maintaining vascular homeostasis is mediated through Mas. ${ }^{10}$ This accumulating evidence suggests that ACE2 in vascular walls may protect from atherosclerosis not only by reducing Ang II signaling but also by promoting Ang 1-7 signaling through Mas. However, it remains unclear whether and how the endogenously expressed ACE2 in vascular cells has a protective role against atherogenic stimulation. Thus, in this study, we used pharmacological inhibition of ACE2 or Mas expressed in vascular cells to investigate the physiological role of endogenous ACE2 and ACE2-mediated Ang 1-7 signaling in atherogenic stimulation by Ang II. 


\section{METHODS}

\section{Cell culture}

A10 cells (rat thoracic VSMCs), human umbilical vein ECs (HUVECs) and THP-1 cells (human acute monocytic leukemia cell line) were obtained from Dainippon Sumitomo Pharma (Osaka, Japan). The growth medium included Dulbecco's modified Eagle's medium (Nissui Pharmaceutical, Tokyo, Japan) supplemented with $10 \%$ fetal bovine serum (JRH Biosciences, Lenexa, KS, USA) and antibiotics (Gibco BRL, Gaithersburg, MD, USA) for A10 cells, endothelial basal medium 2 Bulletkit (Nissui Pharmaceutical, Tokyo, Japan) for HUVECs and RPMI 1640 supplemented with $10 \%$ fetal bovine serum and antibiotics for THP-1 cells. Cell passages $8-14$ for A10 cells and 4-8 for HUVECs were used for all experiments.

\section{Quantitative real-time reverse transcriptase-PCR}

RNA from VSMCs and HUVECs was purified using an RNA isolation protocol (SV Total RNA Isolation System, Promega, Madison, WI, USA). A HighCapacity cDNA Reverse Transcription kit was used according to the manufacturer's instructions (Applied Biosystems, Foster City, CA, USA) to synthesize complementary DNA. Quantitative PCR was carried out using real-time detection technology and analyzed on a model 7900 Sequence Detector (Applied Biosystems) with specific primers and fluorescent probes for ACE2 and Mas (TaqMan Gene Expression Assays, Applied Biosystems). Levels of mRNA were compared at various time points after normalization to concurrent $18 \mathrm{~S}$ rRNA amplification.

\section{Protein analysis}

After $24 \mathrm{~h}$ of serum depletion, subconfluent VSMCs and HUVECs were incubated with Ang II $\left(10^{-7} \mathrm{M}\right.$; Bachem Bioscience, King of Prussia, PA, USA) for $10 \mathrm{~min}$ followed by immunoblot assays for phosphorylated ERK 1/2. In certain experiments, cells were pretreated with either Ang $1-7\left(10^{-7} \mathrm{M}\right.$; Bachem Bioscience), an ARB; olmesartan ( $10^{-6} \mathrm{M}$; Sankyo Laboratories, Rahway, Japan), an ACE2 inhibitor; DX600 (10 ${ }^{-6} \mathrm{M}$; Phoenix Pharmaceuticals, Belmont, CA, USA), a Mas receptor antagonist; D-Ala7-Ang 1-7 (D-Ala; $10^{-5} \mathrm{M}$; Bachem Bioscience) or a combination for $10-20 \mathrm{~min}$ before the application of Ang II. The phosphorylation state of ERK 1/2 was measured by western blotting. Cells were lysed in a buffer consisting of $62.5 \mathrm{~mm}$ Tris- $\mathrm{HCl}$ (pH 6.8), $2 \% \mathrm{w} / \mathrm{v}$ sodium dodecyl sulfate, $10 \%$ glycerol, $50 \mathrm{mM}$ DTT and $0.1 \%$ $\mathrm{w} / \mathrm{v}$ bromophenol blue. The lysate was then sonicated for $5 \mathrm{~s}$ and boiled for $5 \mathrm{~min}$, followed by centrifugation at $12000 \mathrm{~g}$ for $5 \mathrm{~min}$ to remove insoluble debris. Proteins were electrophoretically transferred to nitrocellulose filters. The filters were blocked in Tris-buffered saline solution containing $1 \%$ Tween 20 and 5\% non-fat dried milk. The filters were washed and incubated with primary antibodies. The primary antibodies used were rabbit polyclonal antiphosphorylated extracellular signal-regulated protein kinase (ERK) antibody and rabbit polyclonal antiphospho ERKs antibody (Cell Signaling Technology, Danvers, MA, USA). Filters were thoroughly washed in Trisbuffered saline solution containing $1 \%$ Tween 20 and incubated with horseradish peroxidase-conjugated anti-rabbit secondary antibody (GE Healthcare Life Sciences, Waukesha, WI, USA). Bands were visualized using an enhanced chemiluminescence system (GE Healthcare Life Sciences).

\section{Cell proliferation assay}

The proliferation of VSMCs stimulated by Ang II was determined by estimating viable cells with the Cell Counting Kit-8 (Dojindo, Japan). VSMCs in $100 \mu \mathrm{l}$ medium were seeded onto a 96-well microplate at 10000 cells per well and were incubated overnight at $37^{\circ} \mathrm{C}$. Then, the cells were treated with $10^{-7} \mathrm{M}$ Ang $1-7$, $10^{-6} \mathrm{M}$ olmesartan, $10^{-6} \mathrm{M}$ DX600, or $10^{-5} \mathrm{M}$ D-Ala or a combination of treatments for $60 \mathrm{~min}$ before the application of Ang II. After incubation for $24 \mathrm{~h}, 10 \mu \mathrm{l}$ of Cell Counting Kit- 8 solution was added to each well, and the plates were further incubated for $4 \mathrm{~h}$ at $37^{\circ} \mathrm{C}$. The absorbance at $450 \mathrm{~nm}$ was measured with an ImmunoReader NJ-2000.

THP-1 preparation and adhesion assay of THP-1 cells to HUVECs Staining of THP-1 cells with PKH67 was carried out according to the manufacturer's instructions (Sigma). Before the application of Ang II, HUVECs were treated with $10^{-7} \mathrm{M}$ Ang $1-7,10^{-6} \mathrm{M}$ olmesartan, $10^{-6} \mathrm{M}$ DX600, or $10^{-5} \mathrm{M}$
D-Ala or a combination of treatments for $60 \mathrm{~min}$. The stained THP- 1 cells and HUVECs were activated by exposure to Ang II $\left(10^{-7} \mathrm{M}\right)$ for $4 \mathrm{~h}$. Then, the THP1 cells were seeded onto confluent HUVECs monolayers. After $10 \mathrm{~min}$, nonadherent cells were washed off three times with phosphate-buffered saline. Finally, we counted adherent THP-1 cells with fluorescence microscopy using two different filters: excitation at $490 \mathrm{~nm}$ and emission at $502 \mathrm{~nm}$.

\section{Statistical analysis}

Data were analyzed with Stat View version 4.51. and are presented as mean \pm s.e.m. Statistical analysis was carried out using analysis of variance with Fisher's post-hoc comparison. A value of $P<0.05$ was considered statistically significant.

\section{RESULTS}

Effects of ACE2 and Ang 1-7 on phosphorylation of ERK 1/2 stimulated by Ang II

We confirmed ACE2 and Mas expression from RNA isolated from VSMCs and HUVECs by real-time PCR, and the expression levels of these genes were not altered by any pharmacological treatment used in this study (data not shown). To clarify the role of endogenous ACE2 and its cleavage product Ang 1-7 in Ang II-induced mitogen-activated protein kinase activation, VSMCs and HUVECs were stimulated by Ang II and were pharmacologically inhibited by AT1 (olmesartan), ACE2 (DX600) and Mas (D-Ala).

In both VSMCs and HUVECs, the stimulation by Ang II caused significant increase in ERK 1/2 phosphorylation, which was blocked by pretreatment with olmesartan (Figures $1 \mathrm{a}$ and $\mathrm{b}$, lanes $\mathrm{B}$ and D). DX600 did not significantly alter the phosphorylation of ERK 1/2 stimulated by Ang II (Figures 1a and b, lane C). However, when AT1 was blocked by olmesartan, the additional treatment with DX600 significantly increased ERK 1/2 activation (Figures la and b, lane E). This effect was blunted in response to Ang 1-7 treatment, and this blunting was diminished by the Mas blockade of D-Ala (Figures 1a and b, lanes $\mathrm{F}$ and $\mathrm{H}$ ). D-Ala partly reversed the inhibitory effect of olmesartan on Ang II-induced ERK 1/2 phosphorylation, suggesting that Ang 1-7-mediated Mas activation might be a negative regulator of Ang II-mediated mitogen-activated protein kinase activation (Figures $1 \mathrm{a}$ and $\mathrm{b}$, lane $\mathrm{G}$ ).

\section{Effects of ACE2 and Ang 1-7 on proliferation of SMCs}

The WST assay was carried out using the Cell Counting Kit- 8 to investigate the involvement of ACE2-Ang 1-7-Mas axis in Ang IImediated VSMCs proliferation. Incubation of VSMCs with Ang II for $24 \mathrm{~h}$ caused a significant increase in the cell numbers compared with the control VSMCs, which was completely inhibited by pretreatment with olmesartan (Figure 2, lanes B and D). Pretreatment with DX600 slightly but significantly accelerated the Ang II-mediated proliferation (Figure 2, lane C).

The inhibition of ACE2 by DX600 partly blunted the inhibitory effect of olmesartan on Ang II-mediated proliferation of SMCs (Figure 2, lane E). This effect of DX600 was reduced by the additional treatment with Ang 1-7, which was blocked by D-Ala (Figure 2, lanes F and $\mathrm{H}$ ). Inhibition of Ang 1-7 signaling by D-Ala partly reversed the inhibitory effect of olmesartan against Ang II-mediated cell proliferation (Figure 2, lane G).

\section{Effects of ACE2 and Ang 1-7 on adhesion of monocyte adhesion to ECs}

We investigated the co-culture of THP-1 cells and HUVECs to evaluate the effects of inflammatory cell adhesion to ECs. The coculture of THP-1 cells and HUVECs after Ang II stimulation caused a significant increase in adherent THP-1 cells compared with control, which was blocked by olmesartan (Figure 3, lanes B and D). 
a
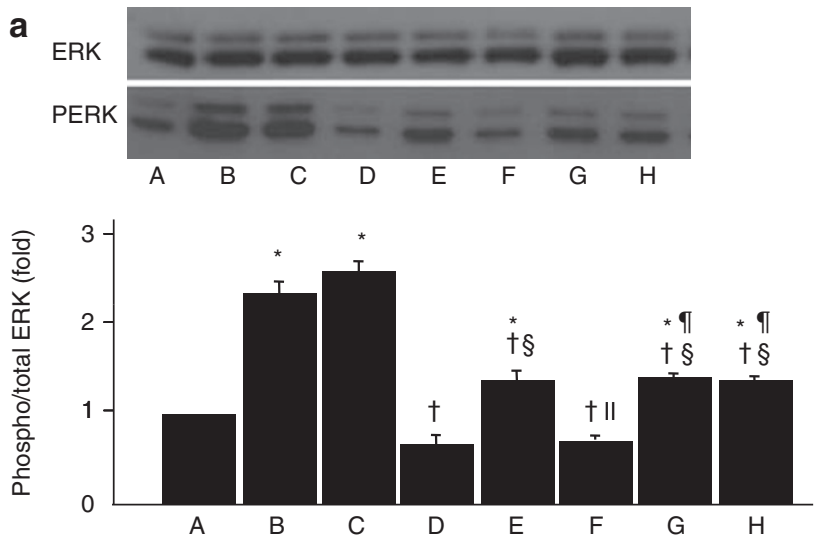

b
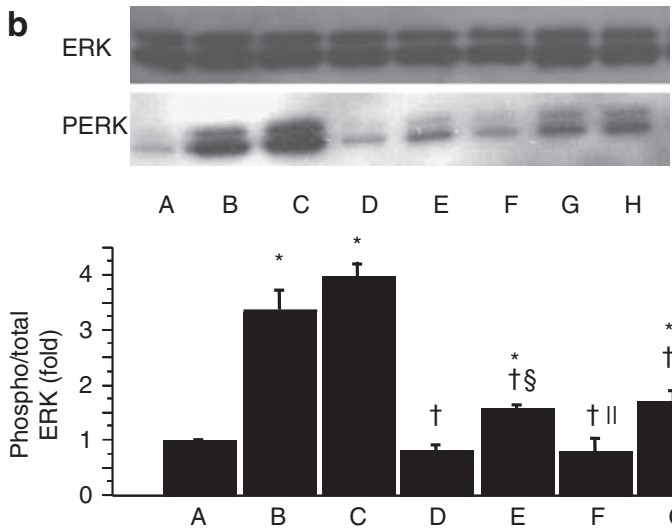
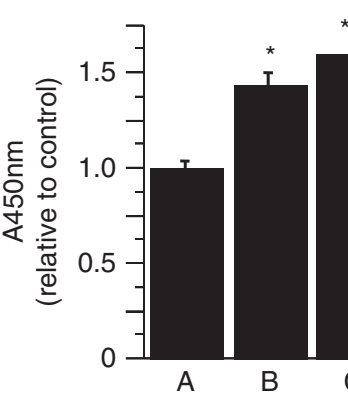

$* \dagger$

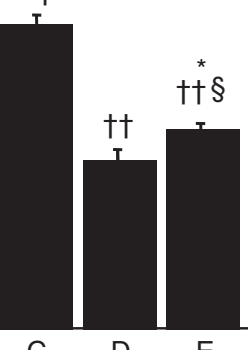

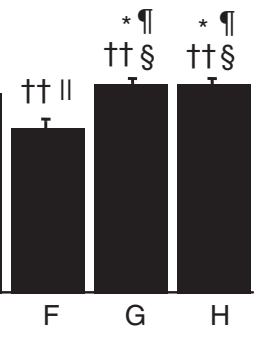

Ang II

\section{DX600}

olmesartan

Ang 1-7

D-Ala

Figure 2 Cell proliferation in VSMCs stimulated by Ang II. The WST assay was carried out using the Cell Counting Kit-8, and absorbance at $450 \mathrm{~nm}$ was measured as an indicator of cell proliferation ( $n=5$, each). ${ }^{*} P<0.01$ vs. lane $\mathrm{A}, \stackrel{\dagger}{\dagger} P<0.05$ vs. Iane $\mathrm{B},{ }^{\dagger} P<0.01$ vs. Iane $\mathrm{B}$, lane $\mathrm{C},{ }^{\circledR} P<0.05$ vs. lane $\mathrm{D}, \| P<0.05$ vs. lane $\mathrm{E}, \uparrow P<0.05$ vs. lane $\mathrm{F}$.

administration of ARB, the inhibition of ACE2 significantly reversed some of the suppressing effects of these Ang II-induced cellular responses. The activation of Ang 1-7-mediated Mas signaling might be involved in the observed effect of ACE2, as indicated by the results of Mas inhibition or Ang 1-7 administration (Figure 4).

The inhibitory effect of Ang 1-7 on Ang II stimulation has been supported by several previous studies using the exogenous administration of the peptide into vascular cells. Freeman et al. ${ }^{8}$ reported that Ang 1-7 inhibited the proliferation of VSMCs induced by Ang II. In human ECs, Ang 1-7 counterregulated the Ang II-induced phosphorylation of c-Src, ERK 1/2 and SHP-2, and the activation of reduced form of nicotinamide adenine dinucleotide phosphate oxidase by Mas receptor. ${ }^{7}$ Thus, it was postulated that endogenous ACE2 in vascular cells might have some protective effects in Ang II-induced vascular injury by producing Ang 1-7 from Ang II. Our results showed that the effect of ACE2 inhibition in the Ang II-induced cellular response was revealed only when the Ang II signaling was reduced by ARB, possibly because of the relatively small amount of Ang 1-7 produced by ACE2 compared with the concentration of the peptide used in previous reports. It has been reported that treatment with ARB increased ACE2 expression in vascular walls; ${ }^{3}$ however, ACE2 mRNA expression was not altered by administration of olmesartan in our study probably because of the short duration of treatment. A long ARB-treatment period might enhance the role of ACE2 by increasing Ang 1-7 production.

When we considered the function of ACE2 in vivo, it should be noted that the catalytic property of ACE2 is not limited to the reninAng system. ${ }^{4}$ In particular, apelin 13 and apelin 36, which are hydrolyzed and rendered inactive by $\mathrm{ACE} 2$, have recently received researchers' attention because these peptides contribute to protection from the development of cardiovascular dysfunction through their receptor apelin. ${ }^{11}$ Thus, the reduction of apelin by ACE2 might counteract its beneficial role in the renin-Ang system in vascular walls. Studies using genetic disruption or pharmacological inhibition of ACE2 in atherosclerotic animals might be useful to determine its physiological role in atherosclerosis.

There are several limitations of this study. First, we used DX600 and D-Ala to inhibit ACE2 and Mas, respectively. Although the concentrations of these agents used in this study were not expected to induce 

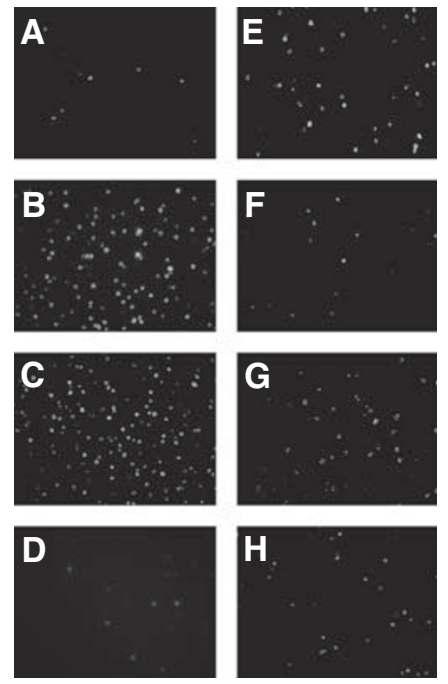

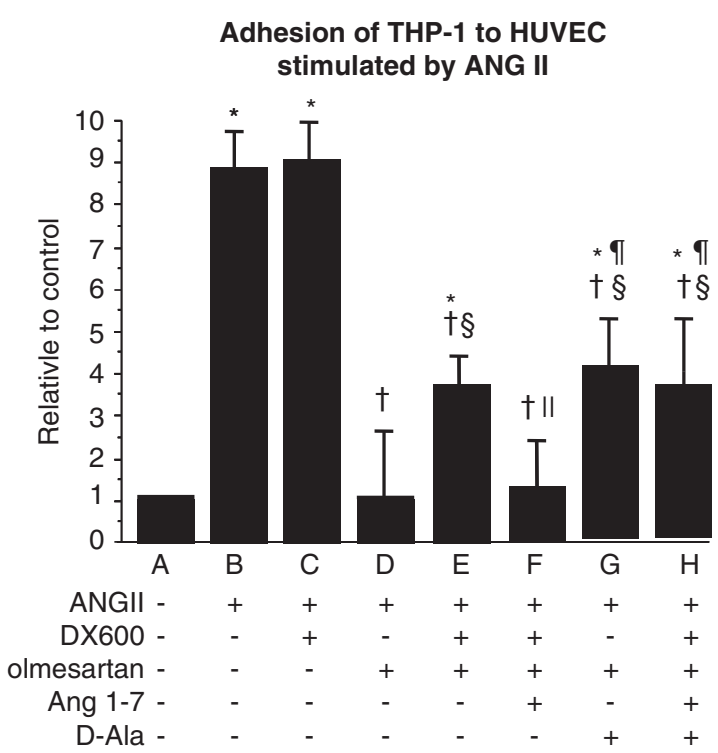

Figure 3 Adhesion of THP-1 cells to HUVECs after stimulation with Ang II. A representative microscopic photograph is shown to the left of the graph (original magnification $\times 400)\left(n=3\right.$, each). ${ }^{*} P<0.01$ vs. lane $\mathrm{A},{ }^{\dagger} P<0.01$ vs. lane $\mathrm{B}$, lane $\mathrm{C},{ }^{\S} P<0.05$ vs. lane $\mathrm{D}, \| P<0.05$ vs. lane $\mathrm{E},{ }^{\uparrow} P<0.05$ vs. lane F.

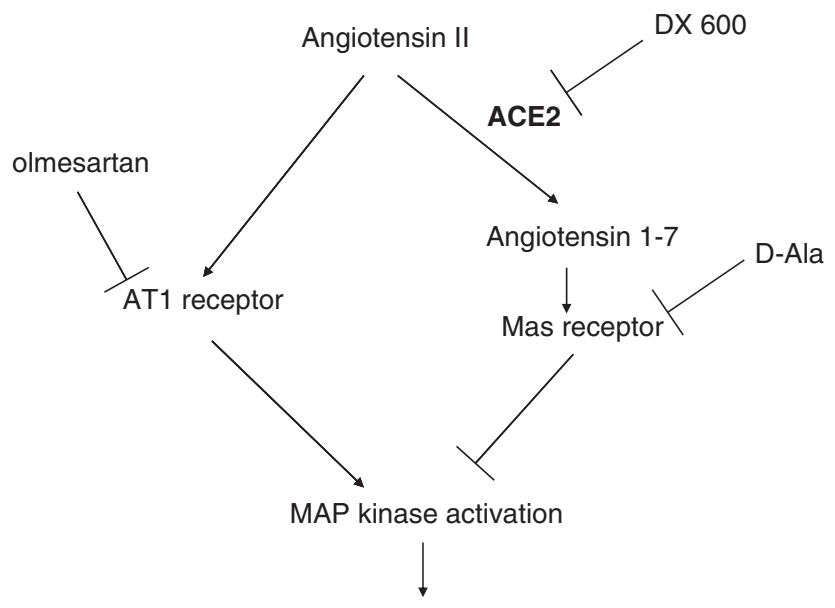

Cell proliferation, adhesion

Figure 4 Summary of the overall mechanism observed in the present study. Endogenous ACE2 in vascular cells might contribute to the inhibition of AT1 receptor-induced mitogen-activated protein kinase activation and subsequent cellular proliferation and adhesion by upregulating the Ang 1-7-Mas pathway.

non-specific effects to cells, a molecular assessment such as one with small interfering RNA needs to be carried out to evaluate the specific effects of these genes. Second, we used A10, HUVECs and THP-1 in this study; the phenotypes of these cell lines differ somewhat from those of primary cells. Freshly isolated cells from animals are more desirable than cell lines for investigating physiological effects.

In conclusion, endogenous ACE2 in vascular cells might counteract Ang II-induced cellular signaling and atherogenic responses by increasing Ang 1-7 at least when ARB is administered. Further investigation would be required to determine whether ACE2 has protective effects against the development of atherosclerosis in vivo.

\section{CONFLICT OF INTEREST}

The authors declare no conflict of interest.

\section{ACKNOWLEDGEMENTS}

We are grateful to Ms Kazuko Iwasa and Ms Eriko Nagata for their technical and secretarial assistance. This study was funded by a Grant-in-Aid (No. 16590684) from the Ministry of Education, Science and Culture and by the Osaka Medical Research Foundation for Incurable Disease.

1 Chiodetti L, Choi S, Barber DL, Schwartz RH. Adaptive tolerance and clonal anergy are distinct biochemical states. J Immunol 2006; 176: 2279-2291.

2 Robinson MJ, Cobb MH. Mitogen-activated protein kinase pathways. Curr Opin Cell Biol 1997; 9: 180-186.

3 Igase M, Strawn WB, Gallagher PE, Geary RL, Ferrario CM. Angiotensin II AT1 receptors regulate ACE2 and angiotensin-(1-7) expression in the aorta of spontaneously hypertensive rats. Am J Physiol Heart Circ Physiol 2005; 289: H1013-H1019.

4 Vickers C, Hales P, Kaushik V, Dick L, Gavin J, Tang J, Godbout K, Parsons T, Baronas E, Hsieh F, Acton S, Patane M, Nichols A, Tummino P. Hydrolysis of biological peptides by human angiotensin-converting enzyme-related carboxypeptidase. J Biol Chem 2002; 277: $14838-14843$

5 Langeveld B, van Gilst WH, Tio RA, Zijlstra F, Roks AJ. Angiotensin-(1-7) attenuates neointimal formation after stent implantation in the rat. Hypertension 2005; 45: 138141.

6 Loot AE, Roks AJ, Henning RH, Tio RA, Suurmeijer AJ, Boomsma F, van Gilst WH. Angiotensin-(1-7) attenuates the development of heart failure after myocardial infarction in rats. Circulation 2002; 105: 1548-1550.

7 Sampaio WO, Henrique de Castro C, Santos RA, Schiffrin EL, Touyz RM. Angiotensin(1-7) counterregulates angiotensin II signaling in human endothelial cells. Hypertension 2007; 50: 1093-1098.

8 Freeman EJ, Chisolm GM, Ferrario CM, Tallant EA. Angiotensin-(1-7) inhibits vascular smooth muscle cell growth. Hypertension 1996; 28: 104-108.

9 Santos RA, Simoes e Silva AC, Maric C, Silva DM, Machado RP, de Buhr I, HeringerWalther S, Pinheiro SV, Lopes MT, Bader M, Mendes EP, Lemos VS, Campagnole-Santos MJ, Schultheiss HP, Speth R, Walther T. Angiotensin-(1-7) is an endogenous ligand for the G protein-coupled receptor Mas. Proc Natl Acad Sci USA 2003; 100: 8258-8263.

10 Xu P, Costa-Goncalves AC, Todiras M, Rabelo LA, Sampaio WO, Moura MM, Santos SS, Luft FC, Bader M, Gross V, Alenina N, Santos RA. Endothelial dysfunction and elevated blood pressure in MAS gene-deleted mice. Hypertension 2008; 51: 574-580.

11 Kalea AZ, Batlle D. Apelin and ACE2 in cardiovascular disease. Curr Opin Investig Drugs 2010; 11: 273-282. 